\title{
金鱼草 $\mathbf{S}$ 核酸酶在大肠杆菌中的表达
}

\author{
杨慧君 薛勇彪* \\ (中国科学院发育生物学研究所, 北京 $100080 .{ }^{*}$ 联系人)
}

摘要 在许多显花植物中, 自交不亲和性由复等位基因构成的被称为 $S$ 位点的单一遗传位点 控制. 在茄科、玄参科和蓄薇科中, 迄今已知的唯一 $S$ 位点编码的产物为一类核酸酶, 称为 $\mathrm{S}$ 核酸酶( S RNases), 为 $S$ 位点在花柱中的产物, 参与自交不亲和性的表达. 作为研究和比较其 三维结构的第一步,在大肠杆菌 (E. coli) 中成功地表达了具有生物活性的金鱼草的 3 种 $S$ 核 酸酶基因 $(S 2, S 4$ 和 $S 5)$ 编码的核酸酶, 结果表明这些基因的表达对 E. coli 的生长没有影响, 为体外大量分离 $\mathrm{S}$ 核酸酶奠定了基础. 还讨论了 $\mathrm{S}$ 核酸酶表达对 E. coli 不致死的可能原因.

\section{关键词 S 核酸酶 自交不亲和性 大肠杆菌 $(E$. coli $)$}

自交不亲和性( self-incompatibility) 是广泛存在于显花植物中的一种抑制自花受精的种内 生殖障碍. 在大多数植物中, 自交不亲和性受控于由复等位基因构成的单一遗传位点, 称为 $S$ 位点 $(S$ locus) . 在茄科、玄参科和蓄薇科中, 迄今已知的唯一 $S$ 位点编码的产物为一类核酸 酶, 即 $\mathrm{S}$ 核酸酶( S RNases)。已有的实验证据表明, $\mathrm{S}$ 核酸酶在花柱中的表达控制自交不亲和 性, 但与 $S$ 位点在花粉中的产物无关, 后者可能是一个不同于 $\mathrm{S}$ 核酸酶的因子 ${ }^{[1]}$. 与 $\mathrm{S}$ 核酸酶 同源的基因分布于整个生物界, 组成所谓的 $\mathrm{S}$ 核酸酶超级家族( superfamily). 除已知 $\mathrm{S}$ 核酸酶 参与自交不亲和反应外, 该家族其他成员的确切生物学功能还不清楚, 可能与防御和 RNA 代 谢等有关 ${ }^{[1]}$.

显花植物 $\mathrm{S}$ 核酸酶的晶体结构目前还未见报道. 无疑, $\mathrm{S}$ 核酸酶三维结构的确定将对了 解它如何参与自交不亲和反应的生化和分子机制有极大的帮助. 作为研究 $\mathrm{S}$ 核酸酶结构的第 一步, 我们在大肠杆菌 $(E . c o l i)$ 中成功地表达了具有生物活性的金鱼草的 3 个不同 $S$ 等位基 因 $(S 2, S 4$ 和 $S 5)$ 编码的 $S$ 核酸酶 ${ }^{[2]}$. 除了用杆状病毒表达膨大矮牵牛 (Petunia inflata) 的 S 核 酸酶的报道外 ${ }^{[3]}$, 本工作是首例在 E. coli 中表达有活性的此类蛋白质.

\section{1 材料与方法}

不含引导肽金鱼草 $S$ 核酸酶基因的分离和克隆: 含 $S 2, S 4$ 和 $S 5$ 基因的金鱼草花柱总 RNA 提取, 第 1 条链 cDNA 的合成和 RACE (rapid amplification of cDNA ends) 按文献 [2] 的方法进 行. 所用的 $S$ 核酸酶基因特异的引物如下: $S 2: \mathrm{Y} 118,5^{\prime}$-CCTTTCCAATTTTTGTTCTAC- $3^{\prime}$ 和 G1280, 5-GCTTGCCCCTTTCTCAAG- $3^{\prime} ; 54$ : Y117, 5-CCTGAACTGTTGTTCAA-3 ${ }^{\prime}$ 和 G1224, 5-ACCACCCACCTCCAAATG $-3^{\prime}$ 和 $S 5: \mathrm{Y}^{\mathrm{Y}} 116,5$-TCTTTCAAGTTATTGTTTTA- $3^{\prime}$ 和 G1481, 5-ACAGTAGGAATTTTGGG- $3^{\prime}$ ). 5 引物( Y118, Y117 和 Y116) 的设计使其正好位于引导肽之后; $3^{\prime}$ 引物则位 于 $S$ 核酸酶基因的终止子之后. cDNA 扩增产物经 Wizard PCR Preps Kit (Promega) 纯化后克隆 进 pGEM-T( Promega) 载体中, 获得 pGEM-S2, pGEM-S4 和 pGEM-S5. 为了避免 PCR(polymerase chain reaction) 产生的碱基错配, 这些克隆所含的 cDNA 序列全部用 ABI 377 测序仪 (PE Applied Biosystems) 做 DNA 序列测定, 结果证明它们与发表的序列一致 ${ }^{[2]}$. 分别用 $N c o$ iv/Sal iv双酶 切, 获得含 $S$ 核酸酶基因的 cDNA 片段, 然后克隆于表达载体 $\mathrm{pET}-15 \mathrm{~b}$ 的 $N c o \mathrm{iv} / X h o$ iv位点, 再 
转化进 E. coli BL21 (Novagen) 中, 分别产生 pET-S2, pET-S4 和 pET-S5.

$\mathrm{S}$ 核酸酶在大肠杆菌的表达和分析: 为了 诱导 $S$ 核酸酶基因的表达, pET-S2, pET-S4 和 pET-S5 分别转化到含有 T7 RNA 聚合酶的 E. coli BL21(DE3) (Novagen)。阳性克隆在 LB 液体培养基中生长到 $A_{600 \mathrm{~mm}}$ 为 0.6 之后, 加 $1 \mathrm{mmol} / \mathrm{L}$ 的异丙基 $-\beta$-硫代半乳糖苗 (IPTG) 诱 导一定时间, 然后提取 E. coli 总蛋白进行 $12 \%$ SDS-PAGE ( sodium dodecyl sulfate-polyacrylamide gel electrophoresis) 分析, 考马斯亮蓝染色检测 蛋白和核酸酶的活性染色按文献 [ 4] 的方法进 行, 即蛋白经 SDS-PAGE 分离之后, 其中 PAG 含 $0.3 \mathrm{mg} / \mathrm{mL}$ 的 rRNA (Type (10) Sigma), 用 Tris-HC1 及异丙醇洗脱恢复蛋白活性, 经亚苯 胺蓝- $O$ 染色, 具有核酸酶活性蛋白质的部位由 于 RNA 降解产生亮斑.

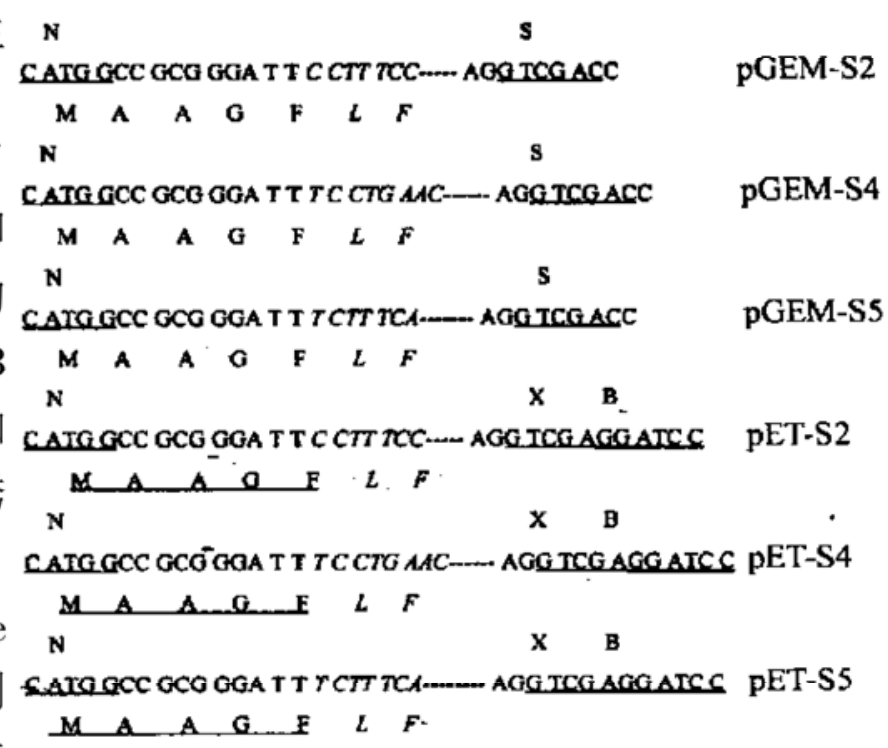

图 $1 \mathrm{~S}$ 核酸酶 5 端序列和表达载体的构建 斜体表示 $\mathrm{S}$ 核酸酶编码的氨基酸, 加底线的氨基酸为克隆 过程中引入. 虚线表示略去的 S 核酸酶和部分载体多克隆 位点区的序列．其余载体的部分未示. $\mathrm{N}-\mathrm{Nco}$ iv, $\mathrm{S}-$ Sal iv, $\mathrm{X}-\mathrm{Xho}$ iv, B-BamH iv

\section{2 结果与讨论}

\section{$2.1 S$ 核酸酶基因在表达载体中的克隆}

为了在体外表达 $\mathrm{S}$ 核酸酶, 我们将 3 个金鱼草 $\mathrm{S}$ 核酸酶 $\mathrm{cDNA}$ 克隆到了 $E$. coli 的表达载 体 pET-15b. S 核酸酶为胞外分泌蛋白, 含有引导肽. 因此, 在克隆到表达载体之前, 首先去除 了其编码的引导肽. 利用 3 对基因特异的引物, 扩增了不含引导肽的 $S 2, S 4$ 和 $S 5$ 的 $\mathrm{cDNA}$ 片 段, 并将其首先克隆于 pGEM-T 载体上, 获得了 pGEM-S2,-S4 和-S5(图 1). S 核酸酶基因 5 引 物的设计使克隆的 cDNA 编码的氨基酸序列与 pGEM-T 上的 Nco iv位点含的 ATG 位于同一个 表达框, 同时引入了 5 个来自载体序列编码的氨基酸(图 1), 这些克隆所含的 $\mathrm{cDNA}$ 编码推测 的蛋白质的分子量分别为 $24.1,23.8$ 和 $23.9 \mathrm{ku}$. 将来自 pGEM-S2,-S4 和-S5 的 Nco iv/ Sal iv DNA 片段分别亚克隆于表达载体 pET-15b 上的酶切位点 Nco iv/Xho iv之间, 获得 pET-S2, -S4 和-S5( 图 1).

\section{$2.2 S$ 核酸酶基因在大肠杆菌中的表达}

表达载体含有 T7 RNA 聚合酶的启动子驱动 S 核酸酶的表达, 因此, 只有在含 T7 RNA 聚 合酶的宿主中才能表达. 同时, 载体上还具有半乳糖苷 $(l a c)$ 操纵子, 可以用 IPTG 人为诱导外 源基因表达, 避免在克隆过程中表达蛋白对宿主产生毒性的可能性, 将 pET-S2,-S4 和-S5 转化 到含有 T7 RNA 聚合酶的 E. coli 宿主 BL21(DE3), 细胞培养到 $A_{600 \mathrm{~mm}}$ 为 0.6 时, 加入 $1 \mathrm{mmol} / \mathrm{L}$ 的 IPTG 诱导 $3 \mathrm{~h}$ 后, 分析 3 种 $S$ 核酸酶基因的表达. SDS-PAGE 的结果表明 3 种 $S$ 核酸酶基 因均有表达, 这些多肽的分子量与其 cDNA 编码的长度大致对应(图 2). 在没有加入 IPTG 的 条件下却无相应的多肽产生, 而且没有外源基因的 $\mathrm{pET}-15 \mathrm{~b}$ 载体对照无特异多肽的表达( 图

2). 我们还发现, 随着 IPTG 诱导的时间延长, $\mathrm{S}$ 核酸酶的表达量逐渐增加( 数据未示).

为了确定所表达核酸酶是否具有生物学活性, 将 SDS-PAGE 分离后的蛋白质进行核酸酶 
活性染色( 图 3), 结果显示在 3 种 $\mathrm{S}$ 核酸酶产物的位置都出现了一个亮区, 说明大肠杆菌表达 的这些 $\mathrm{S}$ 核酸酶都具有核酸酶活性.

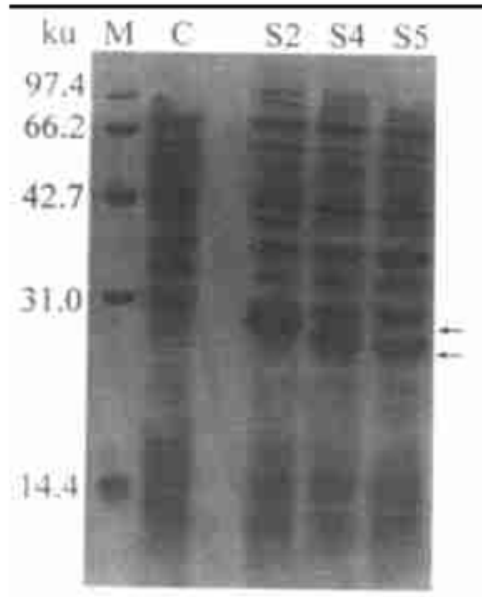

图 $2 \mathrm{~S}$ 核酸酶的体外表达 $\mathrm{M}$ 一一蛋白质分子量标准, C一空白质粒对照, S2 $\longrightarrow$ ET-S2, S4 — $\longrightarrow$ ET-S4, S5 $\longrightarrow$ ET-S5. 箭头所示为表达的 $\mathrm{S}$ 核酸酶多肽

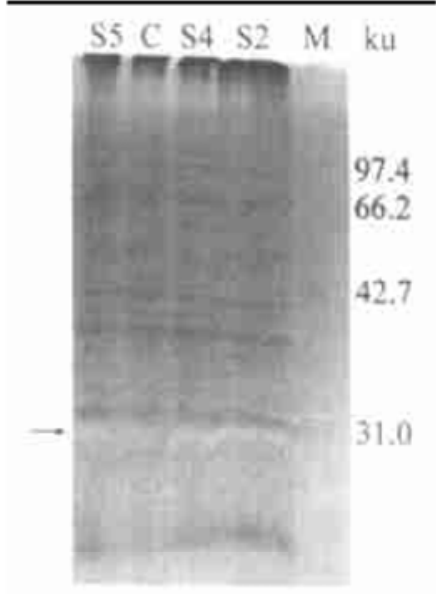

图 3 大肠杆菌中表达的 $\mathrm{S}$ 核酸酶 的活性检测

箭头所示为显示核酸酶活性的部位, 与 $\mathrm{S}$ 核 酸酶多肽的位置一致. M, C, S2, S4, S5 同图 1

为了检测 $\mathrm{S}$ 核酸酶表达是否对大肠杆菌的生长有抑制作用, 含 $\mathrm{pET}-\mathrm{S} 2,-\mathrm{S} 4$ 和-S5 大肠杆 菌培养物加入 IPTG 后, 每隔 $0.5 \mathrm{~h}$ 测定大肠杆菌 $A_{600 \mathrm{~nm}}$ 的值. 结果表明含 3 种 $\mathrm{S}$ 核酸酶基因 的大肠杆菌的生长与对照相比在 $10 \mathrm{~h}$ 内的增长均无差别 (数据未示), 表明 $\mathrm{S}$ 核酸酶的表达并 没有抑制大肠杆菌的生长. 该结果的产生可能有下述几个原因: (1) 虽然 $\mathrm{S}$ 核酸酶在体外复性 后表现出核酸酶活性, 但是在体内由于细胞质环境的原因造成 $\mathrm{S}$ 核酸酶不能正确折叠, 因此对 大肠杆菌的生长不造成影响; (2) 因为大肠杆菌也含有与 $\mathrm{S}$ 核酸酶同源的基因 - RNase I, 它 在细胞质内的表达也不影响细菌的正常生长, 推测可能是由于一种其抑制剂( inhibitor) 的存 在 $^{[5]}$, 后者可能对 $S$ 核酸酶的活性也有抑制作用. 我们将通过研究这些因素进一步探讨 $S$ 核 酸酶的表达对细菌不发生影响的原因. $\mathrm{S}$ 核酸酶在大肠杆菌的成功表达也为我们大量分离并 研究比较该酶的生化特性和晶体结构奠定了基础.

致谢 作者对奇文清对本文提出的意见表示感谢. 本工作为国家自然科学基金( 批准号: 39670387 和 39825103) 和中国科学院的部分基金资助项目.

\section{参 考 文 献}

1 任东路, 张燕生, 薛勇彪. 自交不亲和性的分子机制. 植物科学进展, 1998, 1: 95 106

2 Xue Y, Carpenter R, Dickinson H G, et al. Origin of allelic diversity in Antirrhinum $S$ locus RNases. Plant Cell, 1996, 8: 805 814

3 Mu J, Kao T -H. Expression of two S-robonucleases of Petunia inflata using baculovirus expression system. Bioch Biophy Res Com, 1992, 187: 299 304

4 Blank A, Sugiyama R H, Dekker C A. Activity staining of nucleolytic enzymes after sodium dodecyl sulfate-polyacrylamide gel electrophoresis: use of aqueous isopropanol to remove detergent from gels. Analy Biochem, 1982, 120: 267 275

5 Zhu L, Gangopadhyay T, Padmanabha K P, et al. Escherichia coli rna gene encoding RNase iv: cloning, overexpression, subcellular distribution of the enzyme, and use of an rna deletion to identify additional RNases. J Bacteriol, 1990, 3146 3151

(1999-01-04 收稿) 